\title{
Pharmacological inhibition of sphingosine kinase isoforms alters estrogen receptor signaling in human breast cancer
}

\author{
James W Antoon ${ }^{1}$, William D Meacham', Melyssa R Bratton², Evelyn M Slaughter ${ }^{1}$, \\ Lyndsay V Rhodes ${ }^{2}$, Hasina B Ashe ${ }^{3}$, Thomas E Wiese ${ }^{3}$, Matthew E Burow ${ }^{2}$ \\ and Barbara S Beckman ${ }^{1}$ \\ ${ }^{1}$ Tulane Department of Pharmacology and ${ }^{2}$ Section of Hematology and Medical Oncology, Tulane Department of Medicine, Tulane University School of Medicine, \\ 1430 Tulane Avenue, SL-83, New Orleans, Louisiana 70112, USA \\ ${ }^{3}$ Department of Pharmacy, Xavier University, 7325 Palmetto Street, New Orleans, Louisiana 70125, USA
}

(Correspondence should be addressed to B S Beckman; Email: bbeckman@tulane.edu)

\begin{abstract}
Recently, crosstalk between sphingolipid signaling pathways and steroid hormones has been illuminated as a possible therapeutic target. Sphingosine kinase (SK), the key enzyme metabolizing pro-apoptotic ceramide to pro-survival sphingosine-1-phosphate (S1P), is a promising therapeutic target for solid tumor cancers. In this study, we examined the ability of pharmacological inhibition of S1P formation to block estrogen signaling as a targeted breast cancer therapy. We found that the Sphk1/2 selective inhibitor (SK inhibitor (SKI))-II, blocked breast cancer viability, clonogenic survival and proliferation. Furthermore, SKI-II dose-dependently decreased estrogen-stimulated estrogen response element transcriptional activity and diminished mRNA levels of the estrogen receptor (ER)-regulated genes progesterone receptor and steroid derived factor-1. This inhibitor binds the ER directly in the antagonist ligand-binding domain. Taken together, our results suggest that SKIs have the ability to act as novel ER signaling inhibitors in breast carcinoma.
\end{abstract}

Journal of Molecular Endocrinology (2011) 46, 205-216

\section{Introduction}

Breast cancer is the second leading cause of cancer death in women today, with one in eight women diagnosed in her lifetime, and drug resistance remains the leading cause of breast cancer treatment failure. Approximately $70 \%$ of breast cancer diagnoses are estrogen receptor (ER)-positive, and selective ER modulators (SERMs), such as tamoxifen and fulvestrant, are the first-line therapies for these cancers (Chu \& Anderson 2002, Burstein et al. 2004, Gail et al. 2007, Punglia et al. 2007). Unfortunately, resistance to all endocrine therapies has been documented in both laboratory and clinical experiments, thereby necessitating novel strategies for targeting ER signaling to combat ER-positive breast cancer drug resistance (Dowsett 1996, Kurebayashi 2005).

Over the past several years, much research has been dedicated to the role of lipid signaling in chemotherapy, endocrine therapy and radiation resistance. Alterations in the metabolism of bioactive sphingolipids have been implicated in cancer tumorigenesis and treatment failure (Meacham et al. 2009). Targeting the ceramide (CER)-sphingosine-1-phosphate (S1P) rheostat is of particular interest. The conversion of proapoptotic CER into pro-survival S1P is a proposed 'switch' for cells in an anti-proliferative state to transition to a proliferative state (Cuvillier et al. 1996). The enzyme sphingosine kinase (SK) is the primary regulator for the conversion of CER to S1P, which is a potent inducer of proliferation, tumorigenesis and survival in many solid tumor cancers, including breast and prostate (Taha et al. 2006, Hannun \& Obeid 2008).

Recent studies on the role of SK/S1P in breast cancer suggest this signaling pathway is important in promoting tumorigenesis, endocrine therapy resistance and chemoresistance. SK signaling is a mechanism of estrogen-induced proliferation and $\mathrm{S} 1 \mathrm{P}$ is known to increase levels of circulating estrogen in the system (Doll et al. 2007, Lucki \& Sewer 2008). The role of S1P in endocrine resistance was first characterized by the Xia laboratory, which showed that overexpressing S1P confers tamoxifen resistance to ER-positive MCF-7 cells. Furthermore, it has been shown that growing MCF-7 cells in increasing concentrations of tamoxifen until resistance is established produces an overexpression of SK-1. These results provide evidence that SK/S1P signaling is involved in endocrine drug resistance (Sukocheva et al. 2009). SK can also increase estrogendependent tumorigenesis and promote motility and survival of ER-positive breast cancer (Wang et al. 1999, Nava et al. 2002, Sankala et al. 2007). In a microarray analysis of human breast cancer biopsies, SK mRNA levels directly correlated with a negative prognostic

DOI: 10.1530/JME-10-0116 Online version via http://www.endocrinology-journals.org 
outcome (Ruckhaberle et al. 2008). Given this evidence, the SK/S1P signaling pathway may be a possible therapeutic target to ablate ER-mediated breast cancer proliferation.

Though the CER-S1P has been well characterized over the past several years, there are few pharmacological inhibitors targeting this pathway. The first, and most commonly published, selective inhibitor of SK is SKI-II. This drug has been commercialized and is now readily available from a number of pharmaceutical companies (French et al. 2003). For this reason, SKI-II is the most widely used pharmacological inhibitor of SK and has been utilized in a wide range of studies including those involving cardiology, immunology and oncology (French et al. 2006, Yan et al. 2008, Trifilieff et al. 2009).

SKI-II has been previously studied in the context of lung, brain, breast and prostate cancers (Leroux et al. 2007, Ader et al. 2008, Guillermet-Guibert et al. 2009). In murine models of breast cancer, SKI-II was shown to have an anti-tumor effect with little effect on animal body weight (French et al. 2006). These studies demonstrated that SKI-II has several properties advantageous to drug development, such as high oral bioavailability and limited toxicity (French et al. 2003, 2006). However, there is little in vitro data on SKI-II in ER-positive breast cancer. Furthermore, to date, there are no published studies on the effect of SKI-II on estrogen and ER signaling. Recently, we showed that the Sphk2 selective inhibitor ABC294640 could alter ER signaling in MCF-7 cells (Antoon et al. 2010). In the present study, we demonstrate the ability of SKI-II to abrogate ER signaling, possibly through direct binding to the ER, similar to tamoxifen. SKI-II has potential as a therapeutic agent in breast cancer, as it can diminish survival, viability and proliferation of breast carcinoma cells without similar effects on normal breast epithelial cells. With resistance to first-line drugs such as SERMs on the rise, novel agents targeting ER signaling in human breast cancer are of growing importance.

\section{Materials and methods}

\section{Reagents}

SKI-II (4-[4-(4-chloro-phenyl)-thiazol-2-ylamino]-phenol, CAS 312636-16-1) was purchased from Sigma-Aldrich. Dimethyl sulfoxide (DMSO) and 17ß-estradiol $\left(\mathrm{E}_{2}\right)$ were purchased from Fisher Scientific (Waltham, MA, USA).

\section{Cell culture}

ER-positive MCF-7 cells were cultured as previously described (Antoon et al. 2009). Briefly, the MCF-7 cell line used is a subclone of MCF-7 cells obtained from the
American Type Culture Collection (ATCC, Manassas, VA, USA), which was generously provided by Louise Nutter (University of Minnesota, MN, USA; Burow et al. 1998). The culture flasks were maintained in a tissue culture incubator in a humidified atmosphere of $5 \%$ $\mathrm{CO}_{2}$ and $95 \%$ air at $37^{\circ} \mathrm{C}$. For estrogen studies, cells were washed with PBS three times and grown in phenol red-free DMEM supplemented with $5 \%$ dextran-coated, charcoal-treated fetal bovine serum (5\% CS-FBS) for $72 \mathrm{~h}$ before plating for each particular experiment. MCF10A cells were obtained from ATCC and cultured in mammary epithelial cell medium (Cambrex, San Diego, CA, USA) supplemented with bovine pituitary extract.

\section{Real-time reverse transcription-PCR}

Real-time reverse transcription (RT)-PCR was performed similar to previously reported studies (Boue et al. 2009, Rhodes et al. 2009). In brief, total cellular RNA was extracted using the RNeasy mini column (Qiagen), following the manufacturer's instructions. The concentration of RNA was determined using a u.v. spectrophotometer. RT was performed using the SuperScript First-Strand Synthesis System for RT-PCR (Invitrogen). The level of steroid derived factor-1 (SDF-1) and progesterone receptor $(P G R)$ transcripts was determined using the iQ5 real-time quantitative PCR detection system (Bio-Rad, Inc.). Primers for PCR were designed to span intron-exon junctions to minimize amplification of residual genomic DNA. The primer sequences for PGR, SDF- 1 and $E R$ are (sense and antisense, respectively): PGR (5'-TACCCGCCCTATCTCAACTACC- $3^{\prime} ; 5^{\prime}$-TGCTTCATCCCCACAGATTAAACA- $3^{\prime}$ ), SDF-1 (5'-AGTCAGGTGGTGGGTTAACAG-3' ${ }^{\prime} 5^{\prime}$-AGAGGAGGTGAAGGCAGTGG-3' ${ }^{\prime}$ ), ER (5'-GCGATGGTGGAGATCTTCGA-3'; 5'-CCTCTCCCTGCAGATTCATCA-3' ${ }^{\prime}$ ). PCR mix contained optimal concentrations of primers, cDNA and SYBR Green PCR Master Mix (Bio-Rad Lab.). Quantification and relative gene expression were calculated with internal controls. The ratio between these values obtained provided the relative gene expression levels.

\section{Lipidomics analysis}

Endogenous lipid levels were quantified by mass spectrometry (Lipidomics Core, Medical University of South Carolina), according to published methods (Bielawski et al. 2006). Briefly, cells were collected, fortified with internal standards and extracted with ethyl acetate/isopropyl alcohol. Electrospray ionization, followed by tandem mass spectrometry (ESI/MS/MS) analyses of sphingoid bases, sphingoid base 1-phosphates, CERs and sphingomyelins were performed. 


\section{Western blot analysis}

Protein analysis was performed as described in Burow et al. (1998). Briefly, cells were plated at 50-60\% confluency in $10 \mathrm{~cm}^{2}$ culture flasks in $5 \%$ DMEM for $48 \mathrm{~h}$. Cells were then treated with DMSO or SKI-II $(1-4 \mu \mathrm{M})$ and $10 \%$ DMEM added at 30,60 or $120 \mathrm{~min}$. Following treatment, cells were detached with PBS-EDTA and centrifuged. After removing supernatant, cells were lyzed in 60-100 $\mu$ l lysis buffer (Mammalian Protein Extraction Reagent, MPER, and Halt protease inhibitor, Pierce, Rockford, IL, USA; and PhoSTOP phosphatase inhibitor, Roche). Lysed cells were centrifuged for $10 \mathrm{~min}$ at $12000 \mathrm{~g}$ at $4{ }^{\circ} \mathrm{C}$ to separate protein from cell debris. The supernatants were combined with loading buffer (5\% 2-mercaptoethanol in $4 \times$ LDS Loading Buffer, Invitrogen), boiled for 5 min, and loaded onto a 4-12\% Bis Tris Polyacrylamide Gels (Invitrogen) followed by PAGE at $150 \mathrm{~V}$ for $1 \cdot 25 \mathrm{~h}$. Protein was transferred to nitrocellulose membranes using the iBlot (Invitrogen) transfer unit. Nitrocellulose membranes were blocked in $5 \%$ milk (Bio-Rad Lab.) Tris buffered saline-Tween 20 (TBS-T) for $1 \mathrm{~h}$ at room temperature. Cells were washed briefly with $1 \times$ TBS-T (USB, Cleveland, OH, USA) and primary antibodies were diluted in 5\% BSA (Sigma-Aldrich) TBS-T according to manufacturer's recommended dilutions. Antibodies for tubulin, AKT and phospho-AKT were purchased from Cell Signaling Technology, Inc. (Beverly, MA, USA). Membranes were incubated in primary antibody overnight at $4{ }^{\circ} \mathrm{C}$ with gentle agitation. Secondary infrared conjugated antibodies (LI-COR Biosciences, Lincoln, NE, USA) were diluted in 5\% milk-TBS-T solution at 1:10 000 ratio and the membranes were incubated for $1 \mathrm{~h}$ under gentle agitation at room temperature. Membranes were scanned using the LI-COR Odyssey imager and software (LI-COR Biosciences) to detect total and phosphorylated protein levels in cell lysates. Protein levels were quantified using densitometry analyses.

\section{Clonogenic survival assay}

Colony assays were performed similar to previously published methods (Struckhoff et al. 2004). MCF-7 cells were plated in six-well plates at a density of 1000 cells/ well in full DMEM media. Twenty-four hours later, the cells were treated with SKI-II $(0 \cdot 1-10 \mu \mathrm{M})$ and then monitored for colony growth. Ten days later, the cells were fixed with $3 \%$ glutaraldehyde. Following fixation for $15 \mathrm{~min}$, the plates were washed and stained with a $0.4 \%$ solution of crystal violet in $20 \%$ methanol for 30 min, washed with PBS and dried. Colonies of $\geq 30$ cells were counted as positive. Results were normalized to DMSO vehicle-treated control cells. Statistical analysis of $\mathrm{IC}_{50}$ values were calculated from concentration-response curves, using GraphPad Prism 5.0 (Graphpad Software, San Diego, CA, USA), by applying the equation: $Y=$ Bottom $+($ Top Bottom) $/ 1+10 \log \mathrm{EC}_{50}-X$.

\section{Cell proliferation immunofluorescence assay}

Cells were plated at a density of 10000 cells/well in a 96-well plate containing 10\% DMEM media and allowed to attach overnight. The following day, cells were treated with DMSO or SKI-II for $24 \mathrm{~h}$. At endpoint, cells were fixed using $100 \mu \mathrm{l}$ of $3.7 \%$ formaldehyde in PBS for $10 \mathrm{~min}$. Formaldehyde was removed and the cells were permeabilized using cold methanol for $5 \mathrm{~min}$ at room temperature and washed twice with PBS. One hundred microliters of 3\% FBS in PBS blocking buffer was then added. After $30 \mathrm{~min}$, blocking buffer was removed and cells were incubated for $1 \mathrm{~h}$ with Ki-67 (BD Pharmingen, San Diego, CA, USA) antibody. Cells were then washed with PBS and stained with DAPI nuclear stain for $5 \mathrm{~min}$ before imaging. For staining quantification, numbers of positively stained cells were expressed as a percentage of the total number of cells/field of view/image. The vehicle control was then set to 1 for comparison with SKI-II treatment.

\section{Cell viability assay}

Viability assays were performed as previously described (Struckhoff et al. 2004). Briefly, cells were plated at a density of $7.5 \times 10^{5}$ cells/well in a 96-well plate containing phenol-free DMEM supplemented with $5 \%$ FBS and allowed to attach overnight. Cells were then treated with SKI-II (ranging from $10 \mathrm{nM}$ to $100 \mu \mathrm{M})$ for $24 \mathrm{~h}$. Following treatment, $20 \mu \mathrm{l}$ of 3-(4,5dimethylthiazol-2-yl)-2,5-diphenyltetrazolium bromide (MTT, $5 \mathrm{mg} / \mathrm{ml}$ ) reagent was incubated in each well for $4 \mathrm{~h}$. Cells were lyzed with 20\% SDS in $50 \%$ dimethylformamide. The $\mathrm{pH}$ and absorbances were read on an ELx808 Microtek plate reader (Bio-Tek Instruments, Winooski, VT, USA) at $550 \mathrm{~nm}$, with a reference wavelength of $630 \mathrm{~nm}$.

\section{Estrogen response element-luciferase assay}

As previously described (Boue et al. 2009, Bratton et al. 2009), the cells were seeded in 24-well plates at a density of $5 \times 10^{5}$ cells/well in the same media and allowed to attach overnight. After $18 \mathrm{~h}$, cells were transfected for $5 \mathrm{~h}$ in serum-free DMEM with $300 \mu \mathrm{g}$ pGL2-ERE2XTK-luciferase plasmid, using $6 \mu$ of Effectene (Qiagen) per $\mu \mathrm{g}$ of DNA. After $5 \mathrm{~h}$, the transfection medium was removed and replaced with phenol red-free DMEM supplemented with $5 \%$ CS-FBS containing vehicle, $\mathrm{E}_{2}$, SKI or $\mathrm{E}_{2}+\mathrm{SKI}$, and incubated at $37^{\circ} \mathrm{C}$. After $18 \mathrm{~h}$, the medium was removed and $100 \mu \mathrm{l}$ of lysis buffer was added per well and then incubated for $15 \mathrm{~min}$ at room 
temperature. Cell debris was pelleted by centrifugation at $15000 \mathrm{~g}$ for $5 \mathrm{~min}$. Cell extracts were normalized for protein concentration using reagent according to the manufacturers protocol (Bio-Rad Lab.). Luciferase activity for the cell extracts was determined using luciferase substrate (Promega Corp.) in an Autoluminat Plus luminometer (Berthhold Technologies, Bad Wildbad, Germany).

\section{ER $\alpha$ binding assays}

Receptor-binding assays were performed as previously described (Boue et al. 2009). In this method, recombinant ER is in equilibrium with a fluorescent ligand (ES2) and a concentration of the competitor (SKI-II). The relative displacement of the ES2 is measured as a change in polarization anisotropy. Serial dilutions of competitors (SKI-II and $\mathrm{E}_{2}$ ) were prepared from DMSO stock solutions in screening buffer at the desired concentrations. The ER and ES2 were combined with each competitor aliquot to a final concentration of $2 \mathrm{nM}$ ER and $3 \mathrm{nM}$ ES2, respectively. In addition, both a no-binding control (ER+ES2, equivalent to $0 \%$ competitor inhibition) and a $100 \%$ binding control (only free ES2, no ER, equivalent to $100 \%$ competitor inhibition) were prepared. All competitors and controls were prepared in duplicate within a binding experiment. After a $2 \mathrm{~h}$ incubation at room temperature, the anisotropy value for each sample and control were measured using the Beacon 2000. Anisotropy values were converted to percent inhibition using the following formula: $I_{\%}=\left(A_{0}-A\right) /\left(A_{0}-A_{100}\right) \times 100$, where $I_{\%}$ is the percent inhibition, $A_{0}$ is $0 \%$ inhibition, $A_{100}$ is $100 \%$ inhibition and $A$ represents the observed value. This conversion to percent inhibition makes the data more intuitive and normalizes the experimentto-experiment differences in the range of anisotropy values. The percent inhibition versus competitor concentration curves were analyzed by nonlinear leastsquares curve fitting (Prism 5.0a, GraphPad Software) to yield $\mathrm{IC}_{50}$ values (the concentration of competitor needed to displace half of the bound ligand). To compare binding affinities of the test compounds to those reported in the literature, $\mathrm{IC}_{50}$ values were converted to relative binding affinities (RBA) using $\mathrm{E}_{2}$ as a standard. The $\mathrm{E}_{2} \mathrm{RBA}$ was set equal to 100 $\mathrm{RBA}=\left(\mathrm{IC}_{50} / \mathrm{IC}_{50}\right.$ of $\left.\mathrm{E}_{2}\right) \times 100$.

\section{Molecular modeling}

The structures of SKI-II were converted to unique SMILE strings with ChemDraw (CambridgeSoft, Cambridge, MA, USA) and then converted to 3D structures using MOE 2008.10 (Chemical Computing Group, Montreal, Quebec, Canada). The initial 3D models were then optimized in MOE using the MMFF94 force field with the conjugated gradient method using a termination of $0.005 \mathrm{kcal} / \mathrm{mol}$. The SKI-II model was added to a database containing an optimized model of 4-hydroxytamoxifen (4-OH-Tam). Docking and scoring of SKI-II was performed using the crystal structure of the human ER $\alpha$ ligand-binding domain in complex with 4-OH-Tam (pdb: 3ERT, antagonist configuration) and the docking function of MOE. The A chain, including associated waters, was extracted from the 3ERT crystal structure and then hydrogens were added and optimized for docking with the Protonate 3D function of MOE. The models of SKIII and 4-OH-Tam were then docked into the 3ERT Chain A model with MOE dock using the Triangle Matcher placement (default settings), London dG rescoring 1, Forcefield refinement (default settings) and London $\mathrm{dG}$ rescoring 2. This docking procedure was previously defined in this study as the MOE docking method that produced the best RMSD $(0.758)$ replacement of the 4-OH-Tam model into this crystal structure.

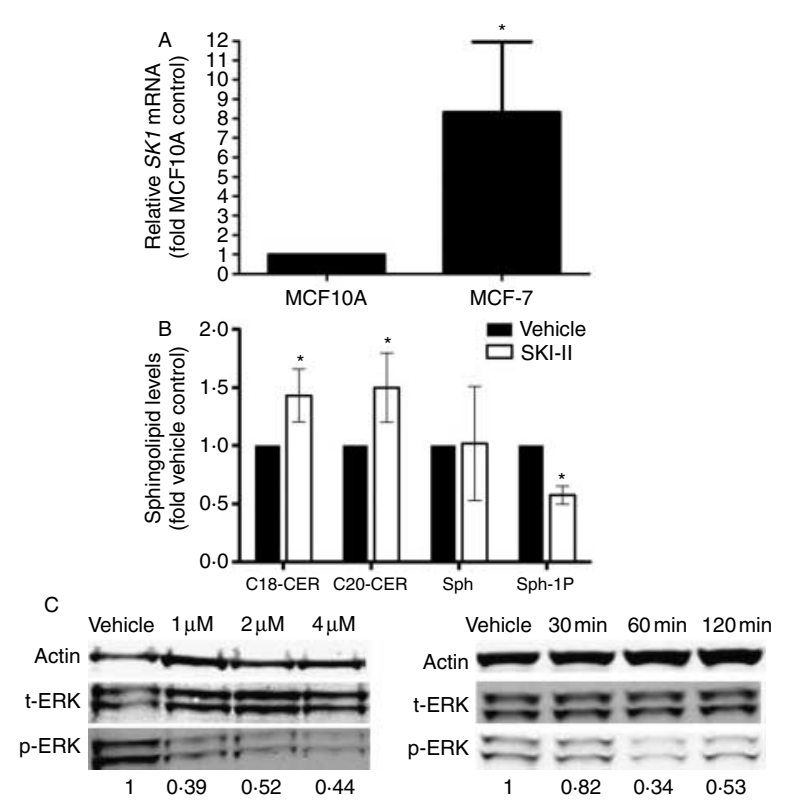

Figure $1 \mathrm{SKI}-\mathrm{II}$ blocks downstream SK signaling. (A) PCR analysis for endogenous expression of $S K 1$. Data are expressed as fold-change relative to MCF10A cell control as normalized to internal $\beta$-actin \pm S.E.M. Data points and error bars represent the mean \pm S.E.M. of three independent experiments. (B) MCF-7 cells were treated with either vehicle control or SKI-II $(10 \mu \mathrm{M})$ for $24 \mathrm{~h}$ and measured for cellular levels of C18-ceramide, C20-ceramide, sphingosine and sphingosine-1-phosphate using ESI/MS/MS. Data points and error bars represent the mean \pm S.E.M. of two independent experiments $\left({ }^{*} P<0 \cdot 05\right)$. (C) MCF-7 cells were treated with either vehicle control or SKI-II analyzed by western blot for phosphorylated and total ERK proteins with densitometry analysis (control set to 1). Phosphorlyated ERK levels were normalized to corresponding $\beta$-tubulin and vehicle control set to 1. Blots are representative of three independent experiments. 


\section{Results}

\section{SKI-II blocks SK/S1P signaling in MCF-7 cells}

It has been previously reported that $S K 1$ mRNA is overexpressed in human breast cancer tissue. Therefore, we chose to examine the expression of $S K 1 \mathrm{mRNA}$ levels in MCF10A cells and compare them to MCF-7 breast cancer cells. MCF10A are nontransformed, noncancerous breast epithelial cell cells commonly used as controls for MCF-7 ductal carcinoma cells. Our RT-PCR results demonstrate that MCF-7 cells have an $8.01 \pm 1.24(P<0.05)$ fold increase in SK1 mRNA expression compared with MCF-10A (Fig. 1A). This overexpression of SK suggests that as cells progress to a cancer phenotype, there is an increase in $S K 1$, which may provide a potential therapeutic target for MCF-7 breast cancer cells.

It was previously reported that SKI-II decreased S1P formation and MAPK signaling in JC murine
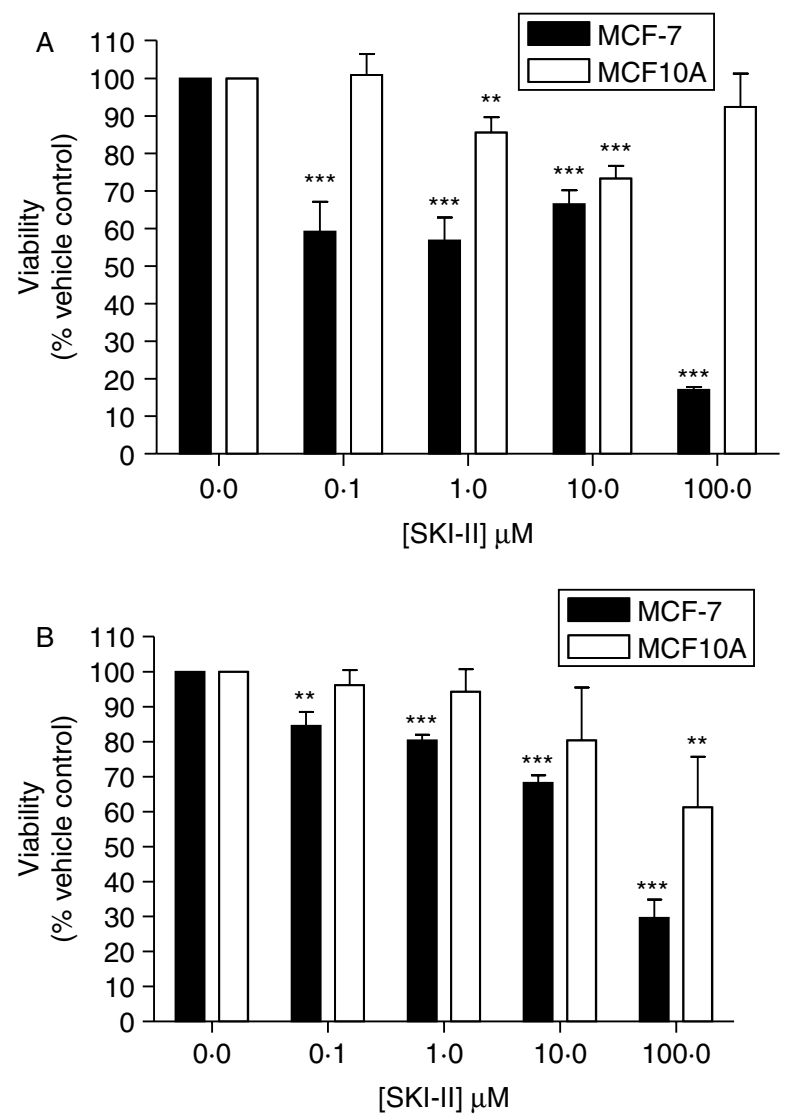

Figure 2 SKI-II decreases MCF-7 viability. MCF10A and MCF-7 cells were treated with SKI-II for (A) $24 \mathrm{~h}$ or (B) $48 \mathrm{~h}$. Cell viability was determined using the MTT assay as described in the Materials and methods section. Data are presented as percent viability of vehicle-treated control cells. Mean values \pm S.E.M. of three different experiments in triplicate are reported $\left({ }^{\star \star \star} P<0.001\right.$, $\left.{ }^{*} P<0.01\right)$.

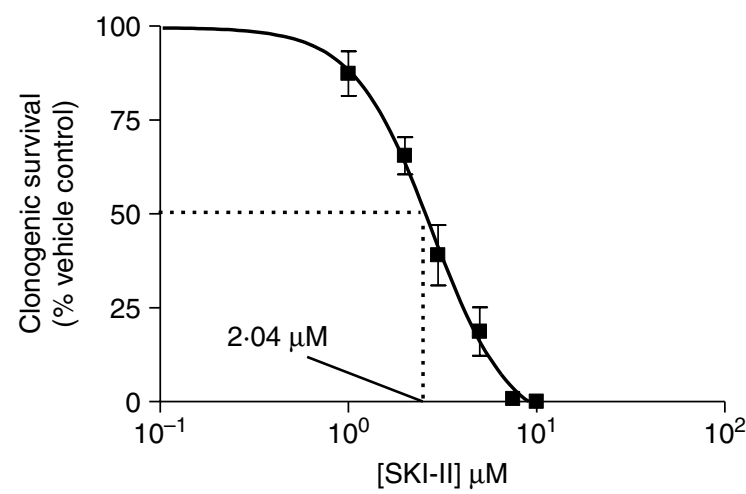

Figure 3 Inhibition of SK diminishes MCF-7 clonogenic survival. MCF-7 cells were plated at 1000 cells/well in six-well plates. Twenty-four hours later, cells were treated with DMSO (vehicle) or SKI-II $(0 \cdot 1-10 \mu \mathrm{M})$ for 14 days. Colonies of $>30$ were counted as positive. Results were normalized to percent clonogenic survival of vehicle control cells. Data represented as mean \pm S.E.M. of three independent experiments.

adenocarcinoma cells (French et al. 2003). However, SKI-II has yet to be examined in ER-positive human breast cancer. Therefore, we examined whether SKI-II could block formation of S1P and increase levels of endogenous long-chain CERs in the ER-positive MCF7 cell system. Since SK blocks the conversion of proapoptotic CER into pro-survival S1P, the concomitant increase in CER and decrease in S1P would be advantageous as a cancer treatment. Using ESI/MS/ MS analyses, we determined levels of S1P, C18-CER and C20-CER in MCF-7 cells with SKI-II treatment. As seen in Fig. 1B, SKI-II treatment diminished formation of S1P by half compared with vehicle control at $24 \mathrm{~h}$, while endogenous levels of C18-CER and C20-CER were increased by 43.5 and $49 \cdot 1 \%$, respectively. There was no statistically significant reduction in the protein levels of the intermediate lipid sphingosine.

We further examined the ability of SKI-II to affect downstream S1P signaling. S1P is known to activate MAPK signaling pathways as a mechanism of its proliferative effects. In particular, the ERK/MAPK pathway is phosphorylated upon stimulation of S1P signaling (Nava et al. 2002). The ERK/MAPK pathway has been shown to be critical in the promotion of breast cancer proliferation and progression to endocrine therapy resistance (Weldon et al. 2002, Geffroy et al. 2005, Thomas et al. 2008). Therefore, we examined the effect of SKI-II on the phosphorylation of the ERK/MAPK protein (Fig. 1C). As expected, SKI-II decreased ERK phosphorylation in both time-course and dose-response analyses. These results provide proof of principle that SKI-II inhibits S1P signaling in the ER-positive MCF-7 breast carcinoma cell system. 


\section{SKI-II decreases breast cancer viability, survival and proliferation in vitro}

Given that SKI-II blocks S1P formation and downstream ERK phosphorylation in breast epithelial cells, we next examined the ability of SKI-II to selectively target normal breast epithelial cells compared with breast cancer cells. Using the MTT assay, we determined the effect of SKI-II on MCF-7 and MCF10A viability both in short- and long-term treatments (Fig. 2). SKI-II diminished MCF-7 cell viability while having little affect on MCF10A cells. At the highest dose of SKI-II tested, $100 \mu \mathrm{M}, 24$ and $48 \mathrm{~h}$ MCF10A cell viability decreased by 7.6 and $32.7 \%(P<0 \cdot 01)$, respectively. In comparison, MCF-7 cells viability decreased by $82.9 \% \quad(P<0.001)$ and $78.6 \%(P<0.01)$ at the 24 and $48 \mathrm{~h}$ time-points. Taken together, these data suggest that SKI-II preferentially affects abnormal breast epithelial cells and is selective for ER-positive breast cancer.

$\mathrm{S} 1 \mathrm{P}$ is known to be a pro-survival mediator and can act in both a paracrine and autocrine manner to promote its effects. There is disagreement in the literature as to whether short-term viability assays accurately reflect chemotherapeutic potential, since
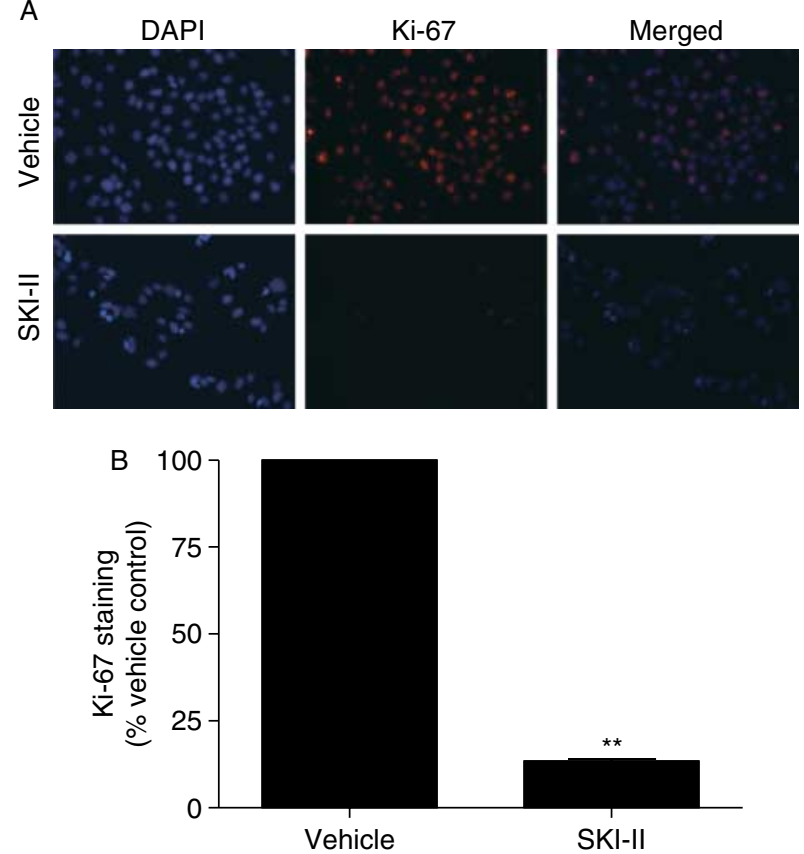

Figure 4 SKI-II blocks MCF-7 proliferation. (A) MCF-7 cells treated with vehicle or SKI-II for $48 \mathrm{~h}$ were fixed using $3.7 \%$ formaldehyde in PBS, permeabilized using cold methanol and incubated with anti-Ki-67 antibody (red). Cells were washed (with PBS) and DAPI (blue) nuclear stained before imaging. Three independent experiments were performed with representative pictures shown here. (B) Quantitation of Ki-67 staining was determined as a percentage of total positive cells/image. The vehicle control was set to 1 for comparison with SKI-II treatment $\left({ }^{\star \star} P<0 \cdot 001\right)$.
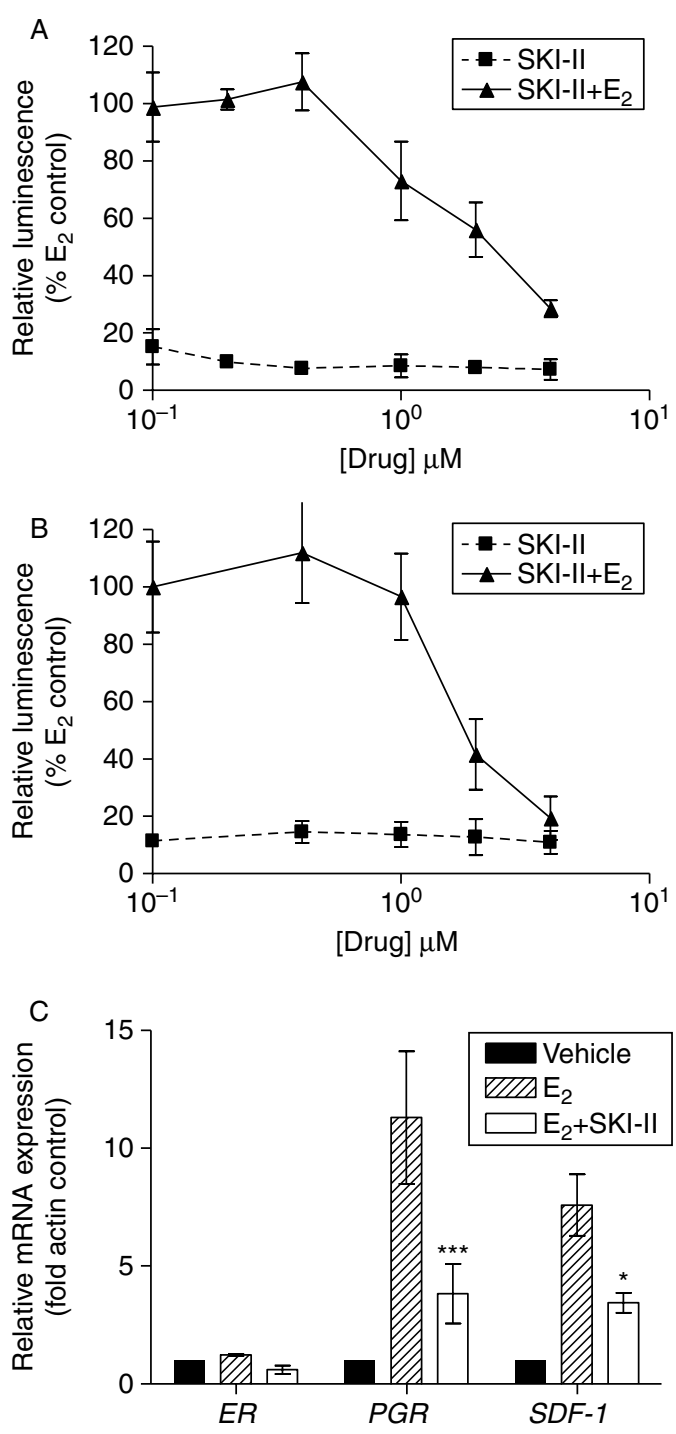

Figure 5 Inhibition of SK decreases estrogen signaling in vitro. HEK293 (A) or MCF-7 (B) cells were transiently transfected with pGI2-ERE2X-TK-luciferase plasmid. HEK293 cells were additionally transfected with pcDNA3.1B-ER $\alpha$ plasmid.

Subsequently, the cells were treated with DMSO (control), estrogen $\left(E_{2}\right)$ or SKI-II. Cells treated with $E_{2}$ were set to 1 . (C) Effect of SKI-II $(10 \mu \mathrm{M})$ on ER $\alpha, P G R$ and SDF-1 gene expression. Statistical analysis was performed comparing treatment groups with $\mathrm{E}_{2}$ control $\left({ }^{\star \star *} P<0 \cdot 001,{ }^{\star} P<0 \cdot 05\right)$.

cancer treatment usually extends over weeks and not hours (Brown \& Wouters 1999). Therefore, we determined the effect of SKI-II on long-term breast cancer clonogenic survival. As seen in Fig. 3, SKI-II dosedependently decreases MCF-7 survival, with an $\mathrm{IC}_{50}$ value of $2.04 \mu \mathrm{M} \quad(P<0 \cdot 01)$. The low-micromolar efficacy of SKI-II indicates the potential of the inhibitor as a breast cancer therapeutic agent.

Given that one of the main actions of S1P is to stimulate proliferation, we next determined whether 
SKI-II could abrogate the growth effects of S1P. The ability to block both proliferation and survival is an advantageous property in any cancer therapy. Using immunofluorescence staining for Ki-67, we found that SKI-II has potent anti-proliferative properties (Fig. 4A). $\mathrm{Ki}-67$ is a protein expressed exclusively in the nucleus during all active phases of the cell cycle and is a known proliferative and prognostic marker for human breast cancer, both in the clinic and in the laboratory (Gaglia et al. 1993, Starborg et al. 1996, Beresford et al. 2006). Treatment with $10 \mu \mathrm{M}$ of SKI-II decreased Ki-67 staining by $86.5 \%(P<0 \cdot 01$; Fig. $4 \mathrm{~B})$. Taken together, these data suggest that pharmacological inhibition of SK has therapeutic potential in treating ER-positive breast cancer.

\section{Inhibition of SK diminishes ER signaling in vitro}

ER signaling is an essential mediator of breast cancer proliferation and tumorigenesis (Fuqua \& Cui 2002, Katzenellenbogen \& Frasor 2004). Given that S1P can promote estrogen-dependent tumorigenesis, as well as increase systemic estrogen levels, we examined the estrogen signaling pathway as a possible mechanism of the potent anti-proliferative and anti-survival properties of SKI-II (Nava et al. 2002, Lucki \& Sewer 2008). The effect of SKI-II on ER transcriptional activity was determined using the estrogen response element (ERE)-luciferase assay. Using the endogenously ER-negative HEK293 cell line, we transiently transfected in both the ER and an ERE-luciferase promoter and treated with increasing concentrations of SKI-II. As seen in Fig. 5A, SKI-II treatment dose-dependently decreased ERE-transcriptional activity. In order to verify that these results occur in an ER-positive system, this experiment was repeated in MCF-7 cells transfected with the same ERE-luciferase construct (Fig. 5B). SKI-II treatment also decreased ERE activity in the MCF-7 cell system, suggesting that this inhibitor can decrease ER activity in vitro.

We further examined whether SKI-II could alter downstream ER-regulated gene expression. SDF-1 (Hall \& Korach 2003) and PGR (Horwitz et al. 1978), two differentially expressed ER-mediated genes, were analyzed using qRT-PCR. Both of these genes are known to transmit the mitogenic and tumorigenic effects of estrogen in breast cancer. As seen in Fig. 5C, treatment with SKI-II blocked estrogenstimulated mRNA expression of SDF-1 and PGR. PGR mRNA expression was decreased by $66 \cdot 1 \%(P<0 \cdot 001)$, while $S D F-1$ expression was decreased by $54 \cdot 6 \%$ $(P<0.05)$ compared with estrogen controls. Taken together, these results indicate that SKI-II can block ER signaling in vitro.

\section{SKI-II directly binds the ER}

Given the decrease in ERE-transcriptional activity, we next determined possible mechanisms of the antiestrogenic effect of SKI-II. Using exogenous competitive binding assays, we found that SKI-II directly binds ER $\alpha$, but not ER $\beta$ (Fig. 6). Interestingly, SKI-II displays an ER $\alpha$ binding IC50 of $85 \cdot 8 \mathrm{nM}(P<0 \cdot 05)$, while estrogen has an $\mathrm{IC}_{50}$ of $0.44 \mathrm{nM}(P<0 \cdot 05)$. This nanomolar $\mathrm{IC}_{50}$ value was surprising compared with the micromolar anti-survival $\mathrm{IC}_{50}$ of this compound. While the tumorigenic and proliferative role of $\mathrm{ER} \alpha$ in the
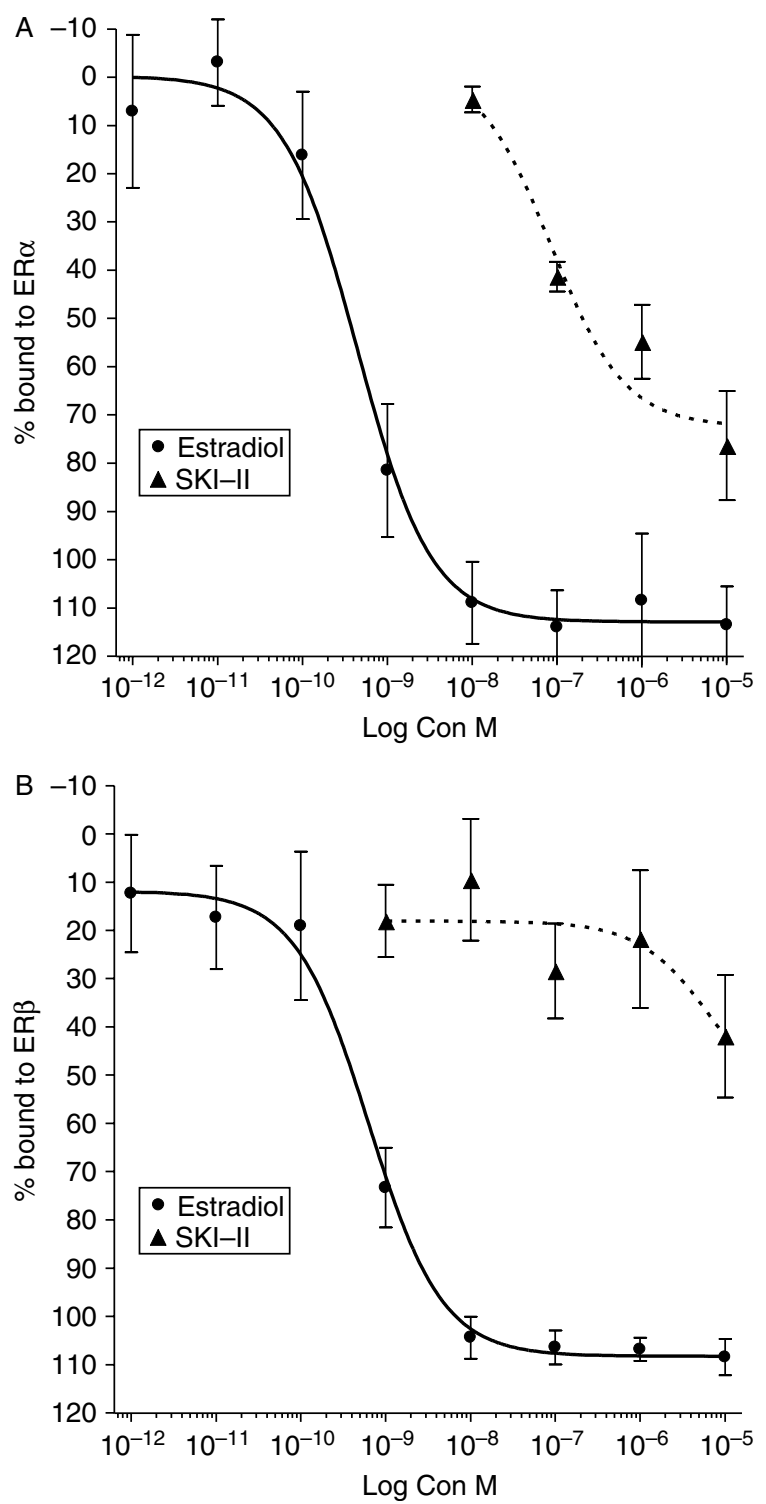

Figure $6 \mathrm{SKI}-\mathrm{II}$ directly binds the ER. Binding affinity of SKI-II was compared with $E_{2}$. Data points and error bars represent the mean \pm S.E.M. of seven independent experiments for SKI-II and $E_{2}$ treatment for each concentration tested. 
promotion of breast cancer is well characterized, it is believed that ER $\beta$ may have opposing effects to that of $\mathrm{ER} \alpha$ (Hartman et al. 2009). Therefore, preferential binding to $\mathrm{ER} \alpha$ may be advantageous in the treatment of ER-positive breast cancer.

Molecular modeling, docking and scoring were utilized in order to better understand the binding of SKI-II to ER $\alpha$ and ER $\beta$. Figure 7 depicts docking simulations of potential SKI-II binding modes in the cavity of ER $\alpha$ (panels A and B) and ER $\beta$ (panel C). The ER-binding cavity is depicted using binding pocket residues, including the critical $\mathrm{H}$-bonding amino acids arginine and glutamate on the right. Two different ER $\alpha$ binding modes of SKI-II were equally scored by the docking simulations (A and B). Pose A utilizes the phenolic ring of the SKI-II to bind the same way that the phenolic group of tamoxifen binds to ER $\alpha$. In this binding mode, the ligand stabilizing H-bonding network between Arg 395, Glu 353 and water can be completed with the phenolic ring of SKI-II. At the same time, the 'rest' of SKI-II occupies the channel leading to the outside of the ER $\alpha$ ligand-binding domain in a similar fashion as the aryl-amino group of 4-hydroxytamoxifen is known to induce the antagonist configuration of ER (Fig. 7A) Pose B utilizes the halogenated phenyl ring of SKI-II to bind ER $\alpha$ in the same binding site location as the phenolic ring of tamoxifen. This halogen may form pseudo H-bonds that are much weaker than those of a hydroxyl group. At the same time, the pose B binding mode of SKI-II places the phenolic $\mathrm{OH}$ in proximity for $\mathrm{H}$-bond interactions with Asp 351 of $\mathrm{ER} \alpha$, the same residue that stabilizes the tertamino of tamoxifen with a charge interaction (Fig. 7B). The docking simulation of SKI-II with ER $\beta$ is shown in panel $\mathrm{C}$ with a binding mode similar to that of the ER $\alpha$ A pose described above and depicted in panel A. In this suggested ER $\beta$ ligand interaction, the phenolic ring binding of SKI-II mimics the phenolic ring interactions of tamoxifen bound to ER $\beta$ (yellow) with the 'rest' of SKI-II occupying the channel leading to the outside of the ER $\beta$ ligand-binding domain as does the aryl-amino group of 4-hydroxy-tamoxifen (Fig. 7C) SKI-II did not present with additional or alternative binding modes in the docking simulations with ER $\beta$.

\section{SKI-II alters ER domain function}

We next set out to determine the mechanism through which SKI-II binding inhibits ER activation. There are two activation regions within $\mathrm{ER} \alpha$, the $\mathrm{AF} 1$ and $\mathrm{AF} 2$ domains. While these two domains usually act in a synergistic manner, they can also be activated independently of each other (Berry et al. 1990, Tzukerman et al. 1994). In certain contexts (such as differential tissue types), AF1 is the dominant activator, while in others, AF2 is the primary modulator of ER function. Estrogen
A

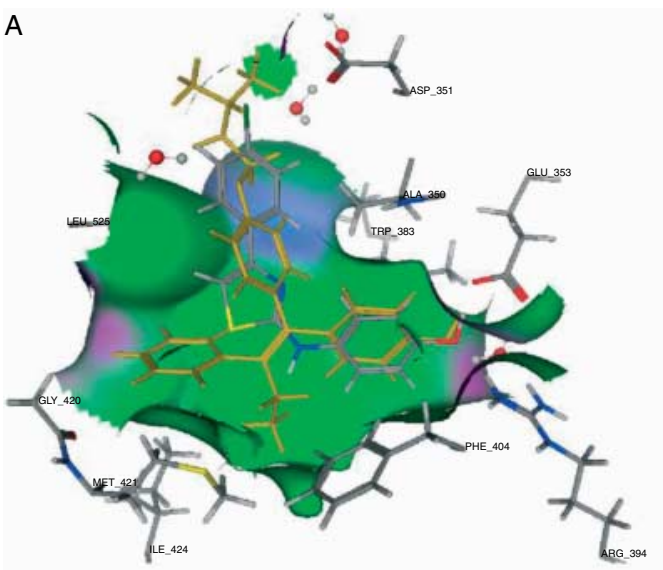

B

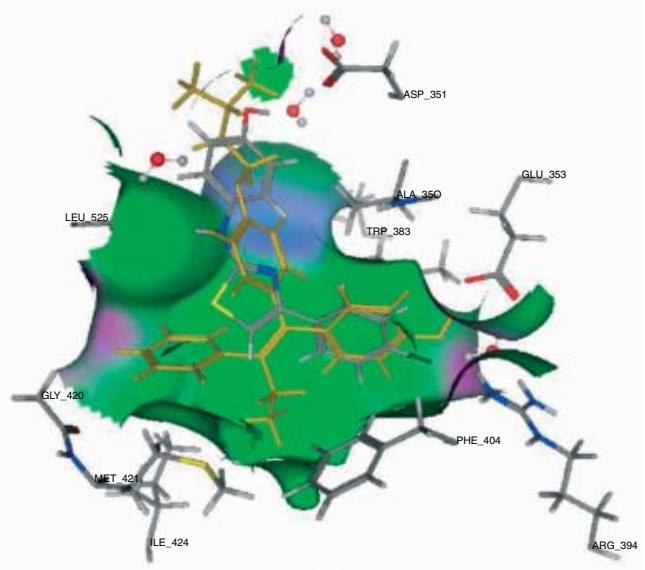

C

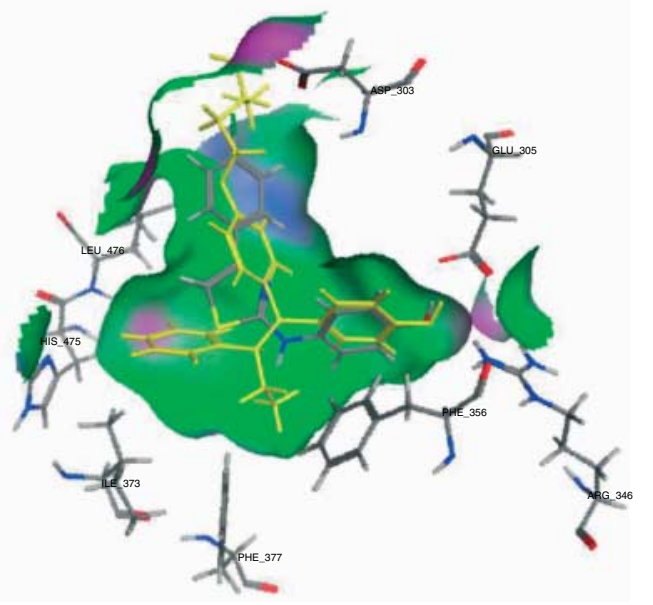

Figure 7 Molecular modeling docking simulations of SKI-II interacting with the ER. SKI-II (atom color) is shown inside the binding cavity of the ER $\alpha$ (panels $A$ and $B$ ) and ER $\beta$ (panel $C$ ) in the context of the 4-OH-Tam (yellow) and key residues of the crystal structures.

activates both domains and is a pure ER agonist in all organ systems. The differential activation of AF1 and AF2 accounts for the tissue-type specific effects of tamoxifen, which blocks only the AF2 domain. Therefore, in tissues where AF2 is dominant, such as the 

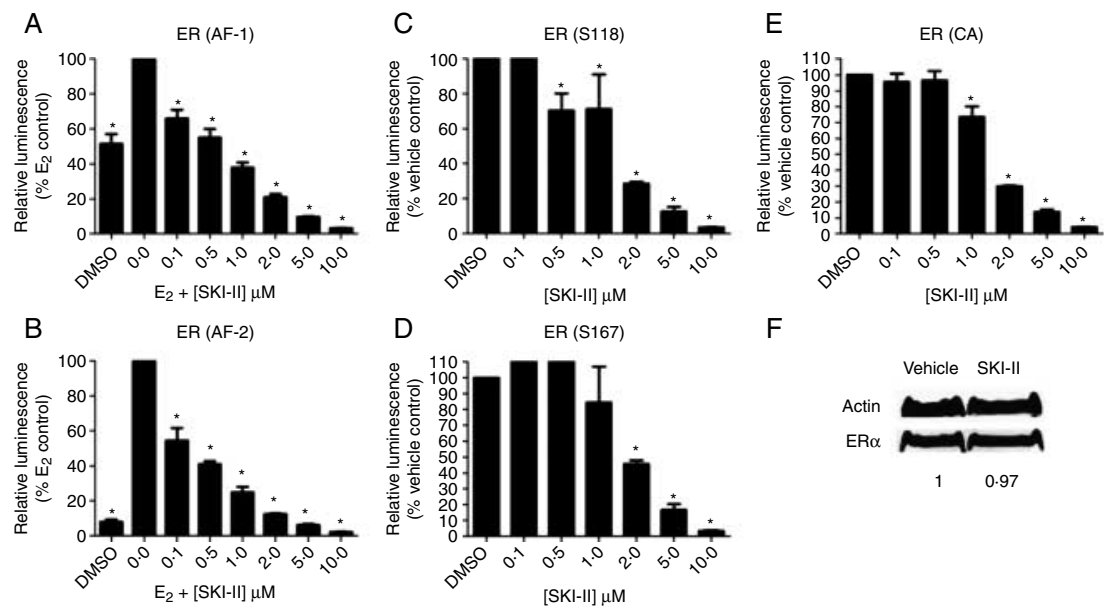

F

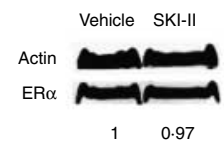

Figure 8 Effect of SKI-II on ER domain function. HEK293 cells were transfected with ER-mutant plasmids containing: (A) AF1 domain knockout, (B) AF2 domain knockout, (C) constitutively active SerS118, (D) constitutively active Ser167 or (E) constitutively active ER. Subsequently, the cells were treated with DMSO (control), estrogen $\left(E_{2}\right)$ or SKI-II. Cells treated with $E_{2}$ were set to 100 . If no $E_{2}$ treatment, DMSO control was set to 100 . Statistical analysis was performed comparing treatment groups with $\mathrm{E}_{2}$ or DMSO control $\left({ }^{\star} P<0.05\right)$. (F) MCF-7 cells were treated with either vehicle control or SKI-II and analyzed by western blot for total ER protein with densitometry analysis (control set to 1). Blots are representative of three independent experiments.

breast, tamoxifen acts an antagonist. In tissues where the AF1 domain is dominant, such as endometrial tissues, tamoxifen acts as an agonist (Hall \& McDonnell 1999). To determine which ER domain SKI-II affects, we utilized two ER mutant models which preferentially inactivate or knockout either AF1 or AF2. As seen in Fig. $8 \mathrm{~A}$ and B, SKI-II dose-dependently blocks ER activity of both inactivated AF1 and knocked-out AF2 models. These results suggest that SKI-II binding to the ER creates a global confirmation change that inhibits both the AF1 and AF2 domains.

SKI-II may inhibit ER signaling by inhibiting the phosphorylation of ER by kinases that are known to be regulated by S1P, such as AKT and ERK. The ER is phosphorylated and activated at serine 118 (S118) and serine 167 (S167) by ERK and AKT, respectively. Therefore, we used two ER mutants, where serine residues 118 and 167 were changed to glutamic acid residues (S118E and S167E), mimicking the phosphorylated state of the residue. As seen in Fig. 8C and D, SKI-II can inhibit ER activity of each mutant, suggesting that SKI-II acts as an ER antagonist independent of ERK and AKT signaling.

In addition to the aforementioned $\mathrm{AF} 1$ and $\mathrm{AF} 2$ domain mutant studies, we used a constitutively activated ER to determine whether SKI-II could inhibit ER activity of a tamoxifen-resistant mutant. The constitutively active Y537S ER mutant is an endogenous mutation found in nature that confers resistance to tamoxifen (Zhang et al. 1997). Using this Y537S ER mutant, we found that SKI-II can inhibit a tamoxifen-resistant ER model, suggesting that SKI-II inhibits the ER in a manner distinct from tamoxifen (Fig. 8E). The fact that SKI-II can inhibit the ER in the setting of tamoxifen resistance makes it an attractive therapeutic option.

\section{Discussion}

Recent research suggests a critical role for bioactive sphingolipids in the promotion, proliferation and tumorigenesis of ER-positive breast cancer (Meacham et al. 2009). S1P, in particular, has been shown to increase systemic levels of circulating estrogen and plays a role in breast cancer survival and endocrine therapy resistance (Lucki \& Sewer 2008, Sukocheva et al. 2009). SK, the main regulator of S1P formation, is an attractive therapeutic target for cancer treatment, though few selective small molecule inhibitors are currently available. The most commonly used inhibitor, SKI-II, is commercially available from multiple sources and has been studied in the context of various cancers, including breast and prostate cancers (French et al. 2006, Leroux et al. 2007).

In this study, we demonstrate, for the first time, the effects of SKI-II on estrogen signaling in ER-positive breast cancer. SKI-II dose-dependently blocks EREtranscriptional activity, both in ER $\alpha$-transfected HEK293 cells and endogenously ER-positive MCF-7 cells. Treatment with SKI-II decreased estrogeninduced transcription of the ER-regulated genes PGR 
and SDF-1. Exogenous competitive binding assays reveal that this inhibitor binds ER $\alpha$, but not ER $\beta$, with an $\mathrm{IC}_{50}$ value in the high nanomolar range. Molecular modeling, docking and scoring analyses suggest that SKI-II binds the antagonist ligand-binding domain of both $\mathrm{ER} \alpha$ and $\mathrm{ER} \beta$ in a similar fashion as the widely used SERM tamoxifen. Taken together, this data suggest a dual mechanism of action for SKII: inhibition of SK and inhibition of ER signaling.

Our findings that pharmacological inhibition of SK can disrupt estrogen signaling are of therapeutic interest in the development of novel breast cancer therapeutics. Nearly, all SERM- and SERD-treated breast cancers ultimately become drug resistant and there are few second-line therapies available, most of which show systemic toxicity (Dowsett 1996, Kurebayashi 2005). Breast cancer chemotherapeutics, such as doxorubicin and paclitaxel, have a myriad of adverse effects and dose-limiting toxicities. The development of new ER targeting agents to treat ER-positive breast cancer is of growing importance. The S1P signaling pathway is known to crosstalk with various elements of the ER signaling cascade. For example, S1P can increase p38/MAPK, ERK/MAPK and PI3K/AKT activity (Baudhuin et al. 2002, 2004). AKT, ERK and p38 are known to phosphorylate the ER independently of ligand and promote endocrine therapy resistance (Weldon et al. 2002, Park et al. 2003, Thomas et al. 2008). Furthermore, S1P can activate the NF- $\kappa \mathrm{B}$ signaling pathway, which is known to mediate superoxide dismutase (SOD). SOD stabilizes the ER complex and enhances ER-DNA binding (Rao et al. 2008). Therefore, the direct binding of SKI-II to the ER may be only one mechanism for the antiestrogenic action of this drug. It is possible there are other secondary mechanisms for this effect as well. Further study is needed to fully understand the effects of SKI-II on ER signaling.

We have further characterized SKI-II as a potential breast cancer therapeutic agent. SKI-II decreases MCF-7 clonogenic survival, with a low $\mathrm{IC}_{50}$ value of $2 \cdot 04 \mu \mathrm{M}$. The inhibitor has potent anti-proliferative properties, with $10 \mu \mathrm{M}$ treatment significantly blocking Ki-67 immunostaining, as well as selective toxicity for abnormal breast epithelia, with little effect on normal breast epithelial cell viability. We have also found that SKI-II can be an effective ER antagonist in the setting of tamoxifen resistance, making SKI-II an attractive therapeutic candidate as an endocrine-resistant breast cancer treatment. It has been previously published that SKI-II possesses high oral bioavailability and little systemic toxicity. Our in vitro results here, combined with previous in vivo studies, indicate that SKI-II is a promising therapeutic agent for the treatment of ER-positive breast cancer.

\section{Declaration of interest}

The authors declare that there is no conflict of interest that could be perceived as prejudicing the impartiality of the research reported.

\section{Funding}

This work was supported by The Center for Bioenvironmental Research at Tulane and Xavier Universities, National Institutes of Health Grant NIDDK and the PhRMA Foundation Paul Calabresi Research Fellowship (J W A).

\section{References}

Ader I, Brizuela L, Bouquerel P, Malavaud B \& Cuvillier O 2008 Sphingosine kinase 1: a new modulator of hypoxia inducible factor $1 \alpha$ during hypoxia in human cancer cells. Cancer Research 68 8635-8642. (doi:10.1158/0008-5472.CAN-08-0917)

Antoon JW, Liu J, Gestaut MM, Burow ME, Beckman BS \& Foroozesh M 2009 Design, synthesis, and biological activity of a family of novel ceramide analogues in chemoresistant breast cancer cells. Journal of Medicinal Chemistry 52 5748-5752. (doi:10.1021/jm9009668)

Antoon JW, White MD, Meacham WD, Slaughter EM, Muir SE, Elliott S, Rhodes LV, Hasina AB, Wiese TE, Smith CD et al. 2010 Anti-estrogenic effects of the novel sphingosine kinase-2 inhibitor ABC294640. Endocrinology 151 5124-5135. (doi:10.1210/en.20100420)

Baudhuin LM, Cristina KL, Lu J \& Xu Y 2002 Akt activation induced by lysophosphatidic acid and sphingosine-1-phosphate requires both mitogen-activated protein kinase kinase and p38 mitogen-activated protein kinase and is cell-line specific. Molecular Pharmacology 62 660-671. (doi:10.1124/mol.62.3.660)

Baudhuin LM, Jiang Y, Zaslavsky A, Ishii I, Chun J \& Xu Y 2004 S1P3mediated Akt activation and cross-talk with platelet-derived growth factor receptor (PDGFR). FASEB Journal 18 341-343.

Beresford MJ, Wilson GD \& Makris A 2006 Measuring proliferation in breast cancer: practicalities and applications. Breast Cancer Research 8 216. (doi:10.1186/bcr1618)

Berry M, Metzger D \& Chambon P 1990 Role of the two activating domains of the oestrogen receptor in the cell-type and promotercontext dependent agonistic activity of the anti-oestrogen 4-hydroxytamoxifen. EMBO Journal 9 2811-2818.

Bielawski J, Szulc ZM, Hannun YA \& Bielawska A 2006 Simultaneous quantitative analysis of bioactive sphingolipids by highperformance liquid chromatography-tandem mass spectrometry. Methods 39 82-91. (doi:10.1016/j.ymeth.2006.05.004)

Boue SM, Tilghman SL, Elliott S, Zimmerman MC, Williams KY, Payton-Stewart F, Miraflor AP, Howell MH, Shih BY, Carter-Wientjes $\mathrm{CH}$ et al. 2009 Identification of the potent phytoestrogen glycinol in elicited soybean (Glycine max). Endocrinology $1502446-2453$. (doi:10.1210/en.2008-1235)

Bratton MR, Frigo DE, Vigh-Conrad KA, Fan D, Wadsworth S, McLachlan JA \& Burow ME 2009 Organochlorine-mediated potentiation of the general coactivator p300 through p38 mitogenactivated protein kinase. Carcinogenesis 30 106-113. (doi:10.1093/ carcin/bgn213)

Brown JM \& Wouters BG 1999 Apoptosis, p53, and tumor cell sensitivity to anticancer agents. Cancer Research 59 1391-1399.

Burow ME, Weldon CB, Tang Y, Navar GL, Krajewski S, Reed JC, Hammond TG, Clejan S \& Beckman BS 1998 Differences in susceptibility to tumor necrosis factor $\alpha$-induced apoptosis among MCF-7 breast cancer cell variants. Cancer Research 58 4940-4946.

Burstein HJ, Polyak K, Wong JS, Lester SC \& Kaelin CM 2004 Ductal carcinoma in situ of the breast. New England Journal of Medicine $\mathbf{3 5 0}$ 1430-1441. (doi:10.1056/NEJMra031301) 
Chu KC \& Anderson WF 2002 Rates for breast cancer characteristics by estrogen and progesterone receptor status in the major racial/ethnic groups. Breast Cancer Research and Treatment $\mathbf{7 4}$ 199-211. (doi:10.1023/A:1016361932220)

Cuvillier O, Pirianov G, Kleuser B, Vanek PG, Coso OA, Gutkind S \& Spiegel S 1996 Suppression of ceramide-mediated programmed cell death by sphingosine-1-phosphate. Nature 381 800-803. (doi:10.1038/381800a0)

Doll F, Pfeilschifter J \& Huwiler A 2007 Prolactin upregulates sphingosine kinase-1 expression and activity in the human breast cancer cell line MCF7 and triggers enhanced proliferation and migration. Endocrine-Related Cancer 14 325-335. (doi:10.1677/ ERC-06-0050)

Dowsett M 1996 Endocrine resistance in advanced breast cancer. Acta Oncologica 35 (Supplement 5) 91-95. (doi:10.3109/ 02841869609083979)

French KJ, Schrecengost RS, Lee BD, Zhuang Y, Smith SN, Eberly JL, Yun JK \& Smith CD 2003 Discovery and evaluation of inhibitors of human sphingosine kinase. Cancer Research 63 5962-5969.

French KJ, Upson JJ, Keller SN, Zhuang Y, Yun JK \& Smith CD 2006 Antitumor activity of sphingosine kinase inhibitors. Journal of Pharmacology and Experimental Therapeutics 318 596-603. (doi:10.1124/jpet.106.101345)

Fuqua SA \& Cui Y 2002 Targeting the estrogen receptor in clinical breast cancer. Breast Disease 15 3-11.

Gaglia P, Bernardi A, Venesio T, Caldarola B, Lauro D, Cappa AP, Calderini P \& Liscia DS 1993 Cell proliferation of breast cancer evaluated by anti-BrdU and anti-Ki-67 antibodies: its prognostic value on short-term recurrences. European Journal of Cancer 29A 1509-1513. (doi:10.1016/0959-8049(93)90284-M)

Gail MH, Anderson WF, Garcia-Closas M \& Sherman ME 2007 Absolute risk models for subtypes of breast cancer. Journal of the National Cancer Institute 99 1657-1659. (doi:10.1093/jnci/djm228)

Geffroy N, Guedin A, Dacquet C \& Lefebvre P 2005 Cell cycle regulation of breast cancer cells through estrogen-induced activities of ERK and Akt protein kinases. Molecular and Cellular Endocrinology 237 11-23. (doi:10.1016/j.mce.2005.04.003)

Guillermet-Guibert J, Davenne L, Pchejetski D, Saint-Laurent N, Brizuela L, Guilbeau-Frugier C, Delisle MB, Cuvillier O, Susini C \& Bousquet C 2009 Targeting the sphingolipid metabolism to defeat pancreatic cancer cell resistance to the chemotherapeutic gemcitabine drug. Molecular Cancer Therapeutics 8 809-820. (doi:10.1158/ 1535-7163.MCT-08-1096)

Hall JM \& Korach KS 2003 Stromal cell-derived factor 1, a novel target of estrogen receptor action, mediates the mitogenic effects of estradiol in ovarian and breast cancer cells. Molecular Endocrinology 17 792-803. (doi:10.1210/me.2002-0438)

Hall JM \& McDonnell DP 1999 The estrogen receptor $\beta$-isoform (ER $\beta$ ) of the human estrogen receptor modulates ER $\alpha$ transcriptional activity and is a key regulator of the cellular response to estrogens and antiestrogens. Endocrinology 140 5566-5578. (doi:10.1210/en. 140.12.5566)

Hannun YA \& Obeid LM 2008 Principles of bioactive lipid signalling: lessons from sphingolipids. Nature Reviews. Molecular Cell Biology 9 139-150. (doi:10.1038/nrm2329)

Hartman J, Strom A \& Gustafsson JA 2009 Estrogen receptor $\beta$ in breast cancer - diagnostic and therapeutic implications. Steroids 74 635-641. (doi:10.1016/j.steroids.2009.02.005)

Horwitz KB, Koseki Y \& McGuire WL 1978 Estrogen control of progesterone receptor in human breast cancer: role of estradiol and antiestrogen. Endocrinology 103 1742-1751. (doi:10.1210/endo103-5-1742)

Katzenellenbogen BS \& Frasor J 2004 Therapeutic targeting in the estrogen receptor hormonal pathway. Seminars in Oncology 31 28-38. (doi:10.1053/j.seminoncol.2004.01.004)

Kurebayashi J 2005 Resistance to endocrine therapy in breast cancer. Cancer Chemotherapy and Pharmacology 56 (Supplement 1) 39-46. (doi:10.1007/s00280-005-0099-z)
Leroux ME, Auzenne E, Evans R, Hail N Jr, Spohn W, Ghosh SC, Farquhar D, McDonnell T \& Klostergaard J 2007 Sphingolipids and the sphingosine kinase inhibitor, SKI II, induce BCL-2-independent apoptosis in human prostatic adenocarcinoma cells. Prostate 67 1699-1717. (doi:10.1002/pros.20645)

Lucki NC \& Sewer MB 2008 Multiple roles for sphingolipids in steroid hormone biosynthesis. Sub-Cellular Biochemistry 49 387-412. (doi:10.1007/978-1-4020-8831-5_15)

Meacham WD, Antoon JW, Burow ME, Struckhoff AP \& Beckman BS 2009 Sphingolipids as determinants of apoptosis and chemoresistance in the MCF-7 cell model system. Experimental Biology and Medicine 234 1253-1263. (doi:10.3181/0902-MR-77)

Nava VE, Hobson JP, Murthy S, Milstien S \& Spiegel S 2002 Sphingosine kinase type 1 promotes estrogen-dependent tumorigenesis of breast cancer MCF-7 cells. Experimental Cell Research 281 115-127. (doi:10.1006/excr.2002.5658)

Park MT, Choi JA, Kim MJ, Um HD, Bae S, Kang CM, Cho CK, Kang S, Chung HY, Lee YS et al. 2003 Suppression of extracellular signalrelated kinase and activation of p38 MAPK are two critical events leading to caspase-8- and mitochondria-mediated cell death in phytosphingosine-treated human cancer cells. Journal of Biological Chemistry 278 50624-50634. (doi:10.1074/jbc.M309011200)

Punglia RS, Morrow M, Winer EP \& Harris JR 2007 Local therapy and survival in breast cancer. New England Journal of Medicine 356 2399-2405. (doi:10.1056/NEJMra065241)

Rao AK, Ziegler YS, McLeod IX, Yates JR \& Nardulli AM 2008 Effects of $\mathrm{Cu} / \mathrm{Zn}$ superoxide dismutase on estrogen responsiveness and oxidative stress in human breast cancer cells. Molecular Endocrinology 22 1113-1124. (doi:10.1210/me.2007-0381)

Rhodes LV, Muir SE, Elliott S, Guillot LM, Antoon JW, Penfornis P, Tilghman SL, Salvo VA, Fonseca JP, Lacey MR et al. 2009 Adult human mesenchymal stem cells enhance breast tumorigenesis and promote hormone independence. Breast Cancer Research and Treatment 121 293-300. (doi:10.1007/s10549-009-0458-2)

Ruckhaberle E, Rody A, Engels K, Gaetje R, von Minckwitz G, Schiffmann S, Grosch S, Geisslinger G, Holtrich U, Karn T et al. 2008 Microarray analysis of altered sphingolipid metabolism reveals prognostic significance of sphingosine kinase 1 in breast cancer. Breast Cancer Research and Treatment 112 41-52. (doi:10.1007/s10549007-9836-9)

Sankala HM, Hait NC, Paugh SW, Shida D, Lepine S, Elmore LW, Dent P, Milstien S \& Spiegel S 2007 Involvement of sphingosine kinase 2 in p53-independent induction of p21 by the chemotherapeutic drug doxorubicin. Cancer Research 67 10466-10474. (doi:10.1158/0008-5472.CAN-07-2090)

Starborg M, Gell K, Brundell E \& Hoog C 1996 The murine Ki-67 cell proliferation antigen accumulates in the nucleolar and heterochromatic regions of interphase cells and at the periphery of the mitotic chromosomes in a process essential for cell cycle progression. Journal of Cell Science 109 143-153.

Struckhoff AP, Bittman R, Burow ME, Clejan S, Elliott S, Hammond T, Tang Y \& Beckman BS 2004 Novel ceramide analogs as potential chemotherapeutic agents in breast cancer. Journal of Pharmacology and Experimental Therapeutics 309 523-532. (doi:10.1124/jpet.103. 062760)

Sukocheva O, Wang L, Verrier E, Vadas MA \& Xia P 2009 Restoring endocrine response in breast cancer cells by inhibition of the sphingosine kinase-1 signaling pathway. Endocrinology 150 4484-4492. (doi:10.1210/en.2009-0391)

Taha TA, Hannun YA \& Obeid LM 2006 Sphingosine kinase: biochemical and cellular regulation and role in disease. Journal of Biochemistry and Molecular Biology 39 113-131.

Thomas RS, Sarwar N, Phoenix F, Coombes RC \& Ali S 2008 Phosphorylation at serines 104 and 106 by Erk1/2 MAPK is important for estrogen receptor- $\alpha$ activity. Journal of Molecular Endocrinology 40 173-184. (doi:10.1677/JME-07-0165)

Trifilieff A, Baur F \& Fozard JR 2009 Role of sphingosine-1-phosphate (S1P) and the S1P(2) receptor in allergen-induced, mast 
cell-dependent contraction of rat lung parenchymal strips. NaunynSchmiedeberg's Archives of Pharmacology 380 303-309. (doi:10.1007/ s00210-009-0438-4)

Tzukerman MT, Esty A, Santiso-Mere D, Danielian P, Parker MG, Stein RB, Pike JW \& McDonnell DP 1994 Human estrogen receptor transactivational capacity is determined by both cellular and promoter context and mediated by two functionally distinct intramolecular regions. Molecular Endocrinology 8 21-30. (doi:10.1210/me.8.1.21)

Wang F, Van Brocklyn JR, Edsall L, Nava VE \& Spiegel S 1999 Sphingosine-1-phosphate inhibits motility of human breast cancer cells independently of cell surface receptors. Cancer Research $\mathbf{5 9}$ $6185-6191$.

Weldon CB, Scandurro AB, Rolfe KW, Clayton JL, Elliott S, Butler NN, Melnik LI, Alam J, McLachlan JA, Jaffe BM et al. 2002 Identification of mitogen-activated protein kinase kinase as a chemoresistant pathway in MCF-7 cells by using gene expression microarray. Surgery 132 293-301. (doi:10.1067/msy.2002.125389)

Yan G, Chen S, You B \& Sun J 2008 Activation of sphingosine kinase-1 mediates induction of endothelial cell proliferation and angiogenesis by epoxyeicosatrienoic acids. Cardiovascular Research $\mathbf{7 8}$ 308-314. (doi:10.1093/cvr/cvn006)

Zhang QX, Borg A, Wolf DM, Oesterreich S \& Fuqua SA 1997 An estrogen receptor mutant with strong hormone-independent activity from a metastatic breast cancer. Cancer Research 57 1244-1249.

Received in final form 1 February 2011

Accepted 14 February 2011

Made available online as an Accepted Preprint 14 February 2011 